\title{
Coronavirus Disease (COVID-19-SARS-CoV-2) and Nutrition: Is Infection in Italy Suggesting a Connection?
}

\author{
Hellas Cena ${ }^{1,2 \star}$ and Marcello Chieppa ${ }^{3,4}$ \\ ${ }^{1}$ Department of Public Health, Experimental and Forensic Medicine-Dietetics and Clinical Nutrition Laboratory, University of \\ Pavia, Pavia, Italy, ${ }^{2}$ Clinical Nutrition and Dietetics Service, Unit of Internal Medicine and Endocrinology, ICS Maugeri IRCCS, \\ Pavia, Italy, ${ }^{3}$ National Institute of Gastroenterology "S. de Bellis", Institute of Research, Castellana Grotte, Italy, ${ }^{4}$ Depatrment \\ of Immunology and Cell Biology, European Biomedical Research Institute of Salerno EBRIS, Salerno, Italy
}

\section{OPEN ACCESS}

Edited by:

Vida Abedi,

Geisinger Health System,

United States

Reviewed by:

Anastasia N. Vlasova,

The Ohio State University,

United States

Alberto Finamore,

Council for Agricultural and

Economics Research (CREA), Italy

*Correspondence:

Hellas Cena

hellas.cena@unipv.it

Specialty section:

This article was submitted to Nutritional Immunology,

a section of the journal

Frontiers in Immunology

Received: 26 March 2020

Accepted: 22 April 2020

Published: 07 May 2020

Citation:

Cena H and Chieppa M (2020)

Coronavirus Disease

(COVID-19-SARS-CoV-2) and

Nutrition: Is Infection in Italy

Suggesting a Connection?

Front. Immunol. 11:944.

doi: 10.3389/fimmu.2020.00944
Novel coronavirus disease (COVID-19) was declared a global pandemic on March 11, 2020. The outbreak first occurred in Wuhan, Hubei, China, in December 2019 and hit Italy heavily in February 2020. Several countries are adopting complete or partial lockdown to contain the growth of COVID-19 infection. These measures may affect people's mental health and well-being but are necessary to avoid spreading the pandemic. There has been a gradual increase in studies exploring prevention and control measures, and we recommend paying close attention to nutrition, which may contribute to modulating some important consequences of COVID-19 infection, as such pro-inflammatory cytokine storm.

Keywords: COVID-19, nutrition, polyphenols, diabetes, incidence, cytokine storm

\section{INTRODUCTION}

Novel coronavirus disease (COVID-19) spread to all regions of Italy on January 31, 2020. The northern region of Lombardy was identified as the center of the two main Italian clusters of cases. On March 11, 2020, the Director-General of the WHO declared COVID-19 a global pandemic as the virus spread rapidly from China to the rest of the world, particularly Europe (1). Currently, the scientific community is sharing potentially useful data to treat patients and protect the population, but the details of COVID-19 infection are still largely unknown, and thus options for risk assessment and pharmacological intervention have only been partially developed.

By March 26, more than 511,603 confirmed cases of COVID-19 had been reported, of which 80,589 were registered in Italy, so far the country with the highest mortality rate (8.215) (2). Four Regions in Italy have reported 59,173 cases of COVID-19, including 34,889 confirmed infections solely in Italy's epicentral Lombardy region, corresponding to more than a third of the total number of infected people in the entire country (Ministry of Health). Italy's mortality rate may be partially explained by the country's relatively higher proportion of older people.

Similarly to what has been observed in China, the most common symptoms were fever, cough, and fatigue (3). Acute respiratory distress syndrome (ARDS) was the main cause of death (4). Older adults and people with chronic illness are most vulnerable to the worst effects of the disease. 
Knowledge about novel COVID-19 is based on a few months of observation and some similarities to Severe Acute Respiratory Syndrome (SARS) and to Middle East Respiratory Syndrome coronavirus (MERS-CoV). Despite the lower case fatality rate than MERS and SARS, COVID-19 has so far proven extremely contagious. Moreover, a significant percentage of those who are infected require hospitalization in an intensive care unit.

Here we speculate on a possible link between nutritional status and COVID-19 mortality, based on data emerging from the Italian National Health System. Furthermore, while waiting for clinical trials to shed light on the clinical efficacy and beneficial effects of antibodies and anti-inflammatory cytokines, we highlight nutritionally derived products that may inhibit the inflammatory cytokine secretion caused by COVID-19 infection.

\section{DIABETES AND COVID-19 INFECTION}

Subjects with diabetes are at risk of infections and have severe disease when infected with a respiratory virus, showing an increased risk of mortality (5).

Data from MERS-CoV, which broke out in 2012 in Saudi Arabia, showed that the severity and length of the pulmonary pathology observed were increased in those affected by type 2 diabetes, which likely deregulates immune response (6). Data on COVID-19 in patients with diabetes is limited at present (5). The Chinese Centre for Disease Control and Prevention published a report of 72,314 cases of COVID-19, showing an increased mortality rate in subjects with diabetes (7).

Diabetes is the most common comorbidity observed in COVID-19-positive deceased patients in Italy after hypertension (8). Available data on pre-existing comorbidities updated on April 9, 2020, and extracted from clinical charts showed hypertension and diabetes before hospitalization in 69.9\% $(n=$ $1,015)$ and $33.8 \%(n=462)$ of COVID-19-positive deceased patients, respectively (9). The same data showed that among 18,366 COVID-19-positive deceased patients, $61.0 \%(n=886)$ presented three or more comorbidities that had been diagnosed before COVID-19 infection, 20.7\% $(n=301)$ had two, $18.8 \%$ ( $n$ $=215)$ had one, and only $3.5 \%(n=51)$ had no pre-existing pathology (9).

Diabetes is prevalent in our population, and most patients with diabetes type II are overweight or affected by obesity (10). However, obesity is not included within the WHO "fiveby-five" framework of non-communicable diseases (NCDs) and risk factors, and data on BMI are not collected in a standardized manner ${ }^{1}$. Unfortunately, we do not yet have weight, height, and waist circumference data for all patients with laboratory-confirmed COVID-19, and, therefore, we cannot disentangle the effects of adiposity on lung function and immune response to viral infection. Excess body weight and increased visceral adiposity are habitually associated with metabolic alterations such as insulin dysregulation, high fasting glucose levels, hyperlipidemia, or systemic hypertension, which

${ }^{1}$ Available online at: https://www.worldobesity.org/training-and-events/events/ wof-webinar-the-collision-of-two-pandemics-covid-19-and-obesity cause dysregulation of the immune system through mediation in various immune, metabolic, and thrombogenic responses. However, the clinical impact of this immune dysregulation on susceptibility to and severity and outcome of viral infections and on lung function is not yet clearly understood $(11,12)$.

Nevertheless, preliminary data from GiViTi (https://giviti. marionegri.it/covid-19/) presented on March 31, 2020, showed a high prevalence of obesity (26\%) and overweight (41\%) in 928 Italian patients, median age 65 years, from 76 different Italian ICUs, confirming evidence available so far in the literature. Recent data on patients with laboratory-confirmed COVID-19 treated at an academic health institution in New York City, the epicenter of the COVID-19 outbreak in the United States, between March 1, 2020, and April 2, 2020, with follow up through April 7, $2020(13,14)$ showed that obesity, after age, was linked to more severe coronavirus cases, with a substantially higher odds ratio than any cardiovascular or pulmonary disease.

Obese and obese-diabetic subjects undergo modifications of the innate and adaptive immune response at different phases, characterized by a state of chronic, and low-grade inflammation and a high basal concentration of several pro-inflammatory cytokines such as alpha-TNF, MCP-1, and IL-6, leading to a defect in innate immunity (14). Recent evidence indicates that obesity not only increases the risk of infection and of complications for the individual but also increases the chance of appearance of a more virulent viral strain, prolonging virus shedding, and eventually increasing the overall mortality rate of an influenza pandemic (15).

Hence, both diabetes and obesity impair the immune response to viral infections like influenza and influenza vaccination through alterations of the cellular immune system (16). Studies so far suggest that diabetics, as well as subjects with obesity, are at a greater risk of hospitalization and increased complications from influenza $(17,18)$. Compared with vaccinated healthy-weight adults, vaccinated obese adults have twice the risk of influenza or influenza-like illness despite equal serological response to vaccination (19). This should be considered one of the challenges to be overcome in vaccine development and/or medications to combat this virulent respiratory virus and prevent future epidemics similar to COVID-19.

\section{HYPERINFLAMMATION IN COVID-19 INFECTED PATIENTS}

Obesity is also associated with chronic low-grade inflammation, dysbiosis, and increased secretion of inflammatory cytokines, including interleukin 6 (IL-6) (20). Elevated plasma levels of pro-inflammatory cytokines are observed in COVID19 infected patients; in particular, IL-6 and ferritin release have been identified as predictors of fatality (21). Several studies focusing on previous outbreaks of severe influenza confirmed that the mortality caused by organ injury could be reduced by immunomodulatory agents $(22,23)$. Currently, a study on the safety and efficacy of Tocilizumab is being conducted (ClinicalTrials.gov Identifier: NCT04317092) (24) to assess its capacity to suppress the virally driven 


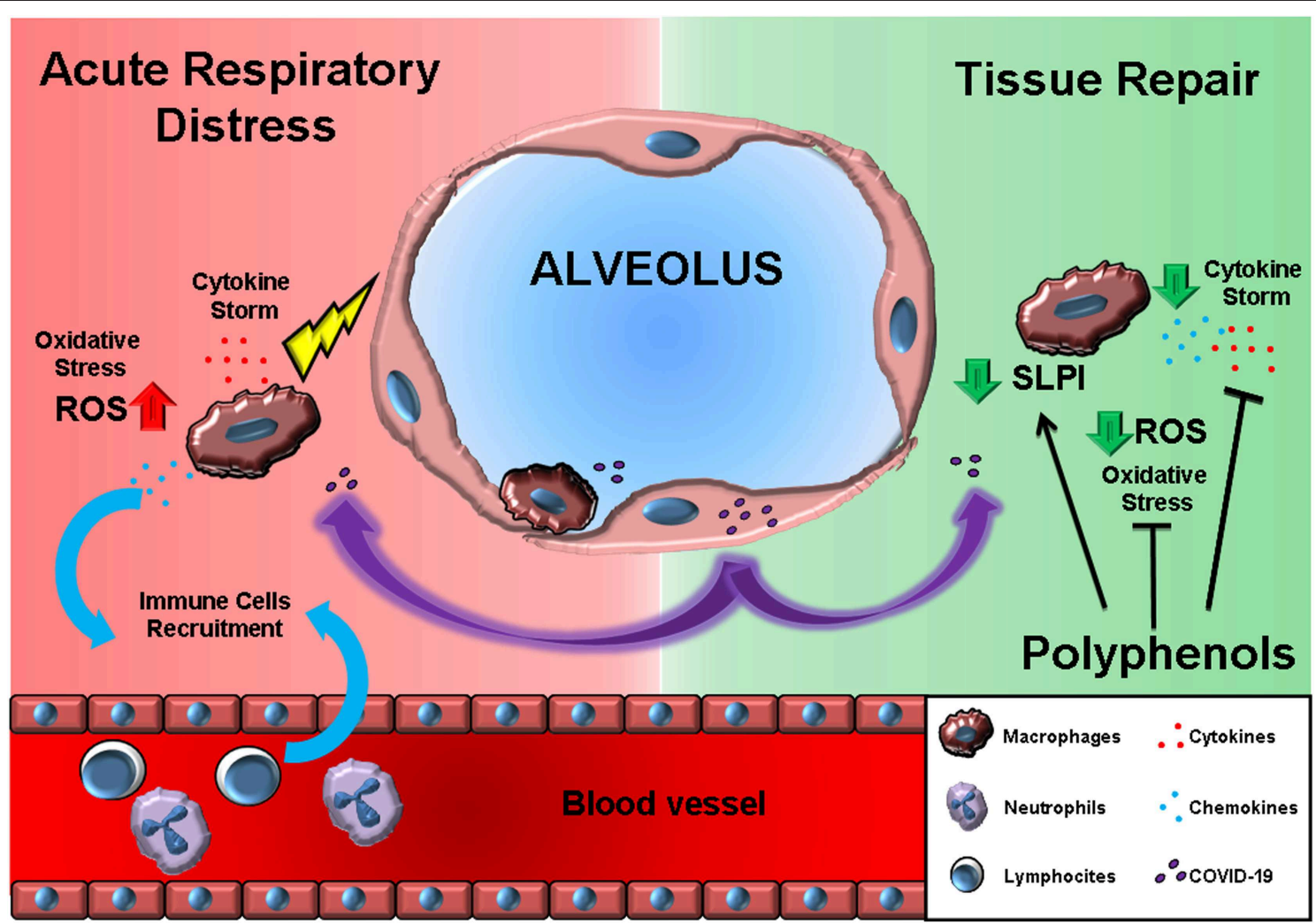

FIGURE 1 | The effects of polyphenols may antagonize the acute respiratory distress induced by macrophage-produced pro-inflammatory cytokine storm. On the left (RED background), COVID-19 infection results in cytokines, and chemokine release by alveolar macrophages. Chemokines further recruit circulating immune cells. On the right (GREEN background), polyphenol exposure can block the immune cell inflammatory program and, at the same time, promote the SLPI release crucial for tissue repair.

hyperinflammation and acute respiratory syndrome caused by COVID-19.

As mentioned previously, COVID-19-related deaths are due to acute respiratory distress syndrome (ARDS); nonetheless, data indicate that the COVID-19 virus is detectable in stool of infected patients, suggesting systemic manifestations (25). In line with this observation, reports indicate abundant expression of ACE2 in absorptive enterocytes of the GI tract (25) and diarrhea as among the most frequent infection symptoms (26). Inflammation of intestinal mucosa may result in increased intestinal permeability with a consequent cascade of events that cause persistent inflammation, worsening the infectionrelated symptoms. Immune homeostasis is a dynamic process maintained by a complex interplay between the gut microbiota and host mucosal immune system (27). Dysbiosis, defined as imbalances in gut microbial species, is now a wellrecognized factor in the pathogenesis of age-associated frailty (28). It is possible that COVID-19 mortality is increased in older patients with comorbidities associated with intestinal dysbiosis, as this could support systemic chronic inflammation in the host. Nonetheless, only future studies will clarify this aspect.

\section{CAN NUTRITIONALLY DERIVED PRODUCTS HELP TO CONTAIN/ALLEVIATE COVID-19-INDUCED INFLAMMATION?}

ACE2-expressing epithelial cells are the primary targets of COVID-19. Similarly to other pulmonary viral infections, following primary exposure, the progeny proliferate in the host cells, which consequently die and release their contents. COVID-19 is now able to infect other cells, including alveolar macrophages (29). Activated or infected immune cells secrete excessive pro-inflammatory cytokines and chemokines, fuelling a vicious circle leading to pulmonary tissue damage. Data accumulating from COVID-19 patients indicate that these patients might have a cytokine storm syndrome, with markedly higher levels of IFN- $\gamma$, CCL-2, CCL-3, TNF, and the aforementioned IL-6 (30-32).

For optimal functioning of the immune system, an adequate nutritional status is required. This is well-acknowledged from evidence linking nutritional deficiencies to the functionality of the immune system (33). Poor nutrition leads to poor immune defense, and it is frequently associated with 


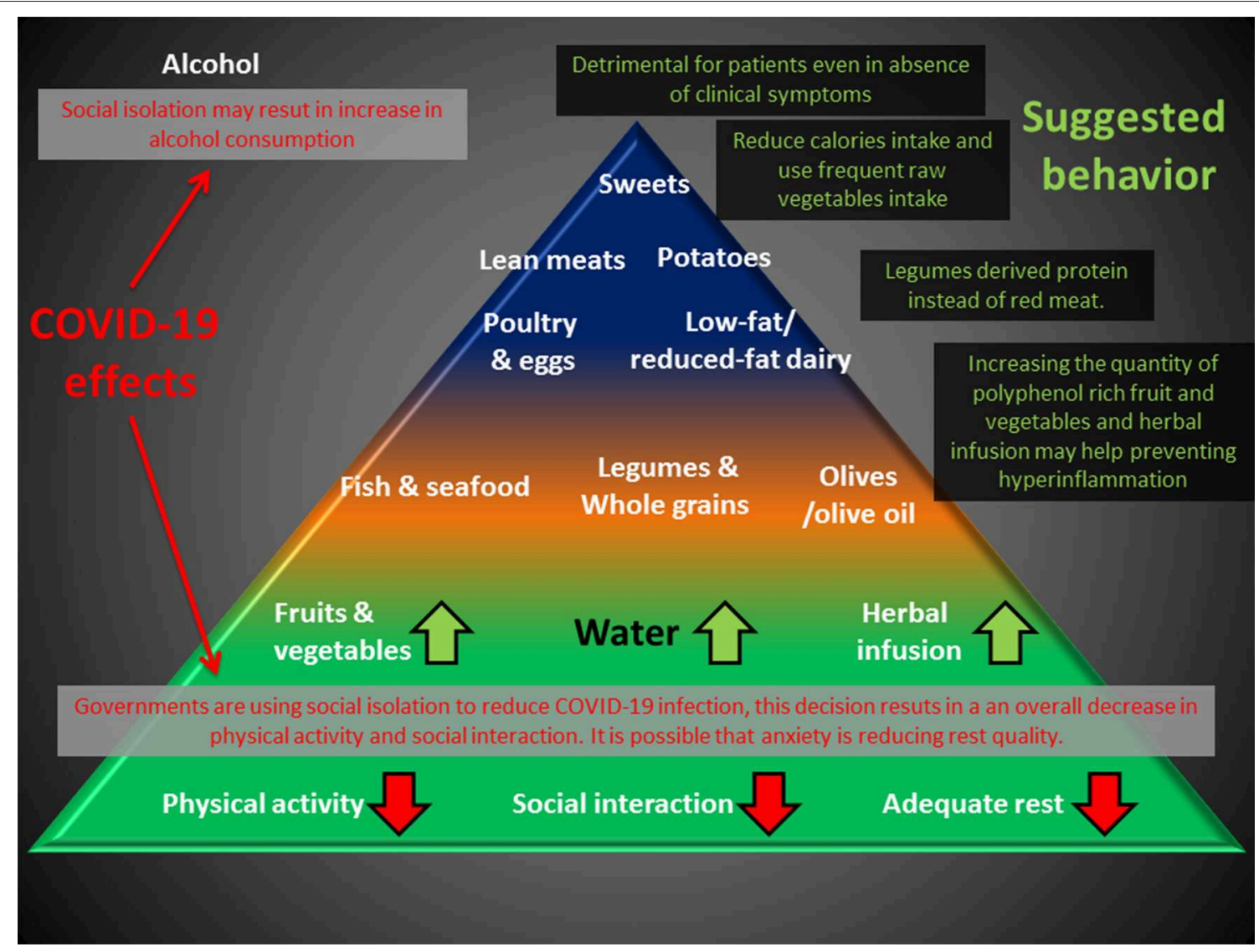

FIGURE 2 | Deleterious effects of the anti-COVID-19 strategy (RED) can impact the base of the Mediterranean diet pyramid. An increase in polyphenol uptake and substitution of meat with legume-derived proteins can help prevent chronic inflammation, and reduction of caloric intake may compensate for the reduction in physical activity. Alcohol consumption is discouraged for healthy people and is detrimental for patients, even in the absence of clinical symptoms.

impaired immunity and increased susceptibility to infection. Nevertheless, nutrient inadequacies and deficiencies in our habitual diet are common (34), and immune function may be improved by restoring nutrients to recommended levels, increasing resistance to infection, and hastening recovery once infected (35).

Furthermore, studies have revealed inadequate micronutrient levels in patients who were hospitalized in the infectious disease department, including thiamine, selenium, zinc, and vitamin B6 deficiencies (36), which were associated with adverse clinical outcomes. Early detection, prevention, and treatment should aim at decreasing the inflammatory response and avoiding the excessive post-inflammatory immune suppression, defined as compensatory response (37), that is observed in many such patients (38).

Various micronutrients are essential for immunocompetence, particularly vitamins A, C, D, E, B2, B6, and B12, folic acid, iron, selenium, and zinc, as well as macronutrients likely omega 3 fatty acids (39) and bioactive components as polyphenols (40).

During recent years, our groups, together with several others worldwide, demonstrated the ability of nutritionally derived bioactive compounds to suppress inflammatory cytokine release (40-43). In particular, the administration of several plant-derived polyphenols to in vitro cultured immune cells suppressed the release of inflammatory cytokines (44). Traditional Chinese herbal medicines for influenza treatment demonstrated potential antiviral activity $(45,46)$. Polyphenol bioavailability has been long discussed, as many have observed health benefits, but few have observed circulating traces of bioactive compounds $(47,48)$. Nonetheless, exposure of the epithelial barrier to a polyphenol-rich environment can efficiently activate local immune suppression and tissue repair mechanisms (49), and systemic benefits may be related to the release of circulating microRNA, defined as small non-coding RNA molecules, with anti-inflammatory effects (50). Indeed, even if orally introduced, bioactive dietary factors induce miRNA synthesis, these are packaged into exosomes and released into the bloodstream to act systemically (51). Dietary factors are emerging as anti-inflammatory miRNA promoters able to regulate metabolic functions, inflammation, and oxidation systemically (52-55). Furthermore, herb extracts may combine antiviral, anti-inflammatory, and antioxidant activity, and tissue-repair properties $(56,57)$ (Figure 1). 
Altogether, these observations suggest that people may benefit from a correct nutritional intake, particularly during this period of uncertainty. Choosing dietary regimes that may potentially work as adjuvants for preventing undesired hyper-inflammation might be particularly useful for patients with mild signs of infection (Figure 2).

\section{DISCUSSION}

General recommendations for healthy adults over 50 years of age observing a period of lockdown and thus with limited options for physical activity should focus on healthy dietary patterns. These can be generally described as those rich in plant-based foods, including fresh fruits and vegetables, soya, nuts, good sources of antioxidants (58), and omega-3 fatty acids (59) and low in saturated fats and trans fats, animalderived proteins, and added/refined sugars (60). Moreover, mild

\section{REFERENCES}

1. WHO. WHO Virtual Press Conference on COVID-19. (2020). Available online at: https://www.who.int/docs/default-source/coronaviruse/transcripts/ who-audio-emergencies-coronavirus-press-conference-full-and-final11mar2020.pdf?sfvrsn=cb432bb3_2 (accessed March 16, 2020)

2. Ruan Q, Yang K, Wang W, Jiang L, Song J. Clinical predictors of mortality due to COVID-19 based on an analysis of data of 150 patients from Wuhan, China. Intensive Care Med. (2020). doi: 10.1007/s00134-020-06028-z. [Epub ahead of print].

3. Coronavirus COVID-19 Global Cases by the Center for Systems Science and Engineering (CSSE) at Johns Hopkins University. Available online at: https://gisanddata.maps.arcgis.com/apps/opsdashboard/index.html\#/ bda7594740fd40299423467b48e9ecf6

4. Wang Z, Yang B, Li Q, Wen L, Zhang R. Clinical features of 69 cases with coronavirus disease 2019 in Wuhan, China. Clin Infect Dis. (2020) pii: ciaa272. doi: 10.1093/cid/ciaa272. [Epub ahead of print].

5. Gupta R, Ghosh A, Singh AK, Misra A. Clinical considerations for patients with diabetes in times of COVID-19 epidemic. Diabetes Metab Syndr. (2020) 14:211-12. doi: 10.1016/j.dsx.2020.03.002

6. Kulcsar KA, Coleman CM, Beck SE, Frieman MB. Comorbid diabetes results in immune dysregulation and enhanced disease severity following MERS-CoV infection. JCI Insight. (2019) 4:131774. doi: 10.1172/jci.insight.131774

7. Wu Z, McGoogan JM. Characteristics of and important lessons from the coronavirus disease 2019 (COVID-19) outbreak in China: summary of a report of 72314 cases from the Chinese center for disease control and prevention. J Am Med Assoc. (2020). doi: 10.1001/jama.2020.2648. [Epub ahead of print].

8. ISS. Characteristics of COVID-19 Patients Dying in Italy Report Based on Available Data on April 9th, 2020. Available online at: https://www.epicentro. iss.it/en/coronavirus/bollettino/Report-COVID-2019_9_april_2020.pdf

9. ISTAT 2015 Statistical Yearbook. Available online at: https://www.istat.it/it/ files//2017/07/REPORT_DIABETE.pdf

10. Peralta GP, Marcon A, Carsin A, Abramson MJ, Accordini S, Amaral AF, et al. Body mass index and weight change are associated with adult lung function trajectories: the prospective ECRHS study. Thorax. (2020) 75:31320. doi: 10.1136/thoraxjnl-2019-213880

11. Marcon A, Corsico A, Cazzoletti L, Bugiani M, Accordini S, Almar E, et al. Body mass index, weight gain, and other determinants of lung function decline in adult asthma. J Allergy Clin Immunol. (2009) 123:106974. doi: 10.1016/j.jaci.2009.01.040

12. Greenberg AS, Obin MS. Obesity and the role of adipose tissue in inflammation and metabolism. Am J Clin Nutr. (2006) 83:461S-5. doi: 10.1093/ajcn/83.2.461S energy restriction is recommended for obese and obese-diabetic patients (15). Most of these dietary targets can be met in our country by means of the well-known and traditionally familiar Mediterranean diet $(61,62)$, which is rich in polyphenols with immune-protective and anti-inflammatory activities, playing an adjuvant role in both prophylaxis and therapy (61). The scientific community is already discussing how to manage future epidemic outbreaks by learning from the current experience (63). Future studies should also focus on the effects of nutrition on immune function, identifying target population subgroups with the most vulnerable immune systems, such as the elderly and those with comorbidities.

\section{AUTHOR CONTRIBUTIONS}

$\mathrm{HC}$ and MC wrote the manuscript.

13. Baffi CW, Wood L, Winnica D, Strollo PJ Jr, Gladwin MT, Que LG, et al. Metabolic syndrome and the lung. Chest. (2016) 149:152534. doi: 10.1016/j.chest.2015.12.034

14. Fernandez C, Manuel A. Obesity, respiratory disease and pulmonary infections. Ann Res Hosp. (2017) 1:38. doi: 10.21037/arh.2017.08.06

15. Petrilli CM, Jones SA, Yang J, Rajagopalan H, O’Donnell LF, Chernyak $\mathrm{Y}$, et al. Factors associated with hospitalization and critical illness among 4,103 patients with Covid-19 disease in New York City. (2020) doi: 10.1101/2020.04.08.20057794

16. Luzi L, Radaelli MG. Influenza and obesity: its odd relationship and the lessons for COVID-19 pandemic. Acta Diabetol. (2020). doi: 10.1007/s00592-020-01522-8. [Epub ahead of print].

17. Sheridan PA, Paich HA, Handy J, Karlsson EA, Schultz-Cherry S, Hudgens M, et al. The antibody response to influenza vaccination is not impaired in type 2 diabetics. Vaccine. (2015) 33:3306-13. doi: 10.1016/j.vaccine.2015.05.043

18. Allard R, Leclerc P, Tremblay C, Tannenbaum TN. Diabetes and the severity of pandemic influenza A (H1N1) infection. Diabetes Care. (2010) 33:14913. doi: $10.2337 / \mathrm{dc} 09-2215$

19. Moser JS, Galindo-Fraga A, Ortiz-Hernández AA, Gu W, Hunsberger S, Galán-Herrera JF, et al. Underweight, overweight, and obesity as independent risk factors for hospitalization in adults and children from influenza and other respiratory viruses. Influenza Other Respir Viruses. (2019) 13:39. doi: 10.1111/irv.12618

20. Green WD, Beck MA. Obesity impairs the adaptive immune response to influenza virus. Ann Am Thorac Soc. (2017) 14(Suppl. 5):S4069. doi: 10.1513/AnnalsATS.201706-447AW

21. Rexrode KM, Pradhan A, Manson JE, Buring JE, Ridker PM. Relationship of total and abdominal adiposity with CRP and IL-6 in women. Ann Epidemiol. (2003) 13:674-82. doi: 10.1016/S1047-2797(03)00053-X

22. Kudo K, Takasaki J, Manabe T, Uryu H, Yamada R, Kuroda E, et al. Systemic corticosteroids and early administration of antiviral agents for pneumonia with acute wheezing due to influenza A(H1N1)pdm09 in Japan. PLoS ONE. (2012) 7:e32280. doi: 10.1371/journal.pone.0032280

23. Zheng BJ, Chan KW, Lin YP, Zhao GY, Chan C, Zhang HJ, et al. Delayed antiviral plus immunomodulator treatment still reduces mortality in mice infected by high inoculum of influenza A/H5N1 virus. Proc Natl Acad Sci USA. (2008) 105:8091-6. doi: 10.1073/pnas.0711942105

24. Chinese Clinical Trial Registry. A Multicenter, Randomized Controlled Trial for the Efficacy and Safety of Tocilizumab in the Treatment of New Coronavirus Pneumonia (COVID-19). (2020). Available online at: http://www.chictr.org. $\mathrm{cn} /$ showprojen.aspx?proj=49409 (accessed March 6, 2020).

25. Zhang $\mathrm{H}$, Kang $\mathrm{Z}$, Gong $\mathrm{H}$, Xu D, Wang J, Li Z, et al. Digestive system is a potential route of COVID-19: an analysis of single-cell 
coexpression pattern of key proteins in viral entry process. Gut Immun. (2020). doi: 10.1136/gutjnl-2020-320953

26. Perlman S. Another decade, another coronavirus. N Engl J Med. (2020) 382:760-2. doi: 10.1056/NEJMe2001126

27. Eri R, Chieppa M. Messages from the inside. the dynamic environment that favors intestinal homeostasis. Front Immunol. (2013) 4:323. doi: $10.3389 /$ fimmu.2013.00323

28. Livshits G, Kalinkovich A. Inflammaging as a common ground for the development and maintenance of sarcopenia, obesity, cardiomyopathy and dysbiosis. Ageing Res Rev. (2019) 56:100980. doi: 10.1016/j.arr.2019.100980

29. Liu Q, Zhou YH, Yang ZQ. The cytokine storm of severe influenza and development of immunomodulatory therapy. Cell Mol Immunol. (2016) 13:310. doi: $10.1038 / \mathrm{cmi} .2015 .74$

30. La Gruta NL, Kedzierska K, Stambas J, Doherty PC. A question of selfpreservation: immunopathology in influenza virus infection. Immunol Cell Biol. (2007) 85:85-92. doi: 10.1038/sj.icb.7100026

31. Mehta PK, McAuley DF, Brown M, Sanchez E, Tattersall RS, Manson JJ, et al. COVID-19: consider cytokine storm syndromes and immunosuppression. Lancet. 395:1033-34. doi: 10.1016/S0140-6736(20)30628-0

32. Huang C, Wang Y, Li X, Ren L, Zhao J, Hu Y, et al. Clinical features of patients infected with 2019 novel coronavirus in Wuhan, China. Lancet. (2020) 395:497-506. doi: 10.1016/S0140-6736(20)30183-5

33. Rijkers GT. Nutrition, immunity and human health. Br J Nutrit. (2013) 114:1329-30. doi: 10.1017/S0007114513002158

34. Bruins MJ, Van Dael P, Eggersdorfer M. The role of nutrients in reducing the risk for noncommunicable diseases during aging. Nutrients. (2019) 11:85. doi: 10.3390/nu11010085

35. Maggini S, Pierre A, Calder PC. Immune function and micronutrient requirements change over the life course. Nutrients. (2018) 10:1531. doi: 10.3390/nu10101531

36. Dizdar OS, Baspinar O, Kocer D, Dursun ZB, Avci D, Karakükcü C, et al. Nutritional risk, micronutrient status and clinical outcomes: a prospective observational study in an infectious disease clinic. Nutrients. (2016) 8:124. doi: 10.3390/nu8030124

37. Gentile LF, Cuenca AG, Efron PA, Ang D, Bihorac A, McKinley BA, et al. Persistent inflammation and immunosuppression: a common syndrome and new horizon for surgical intensive care. J Trauma Acute Care Surg. (2012) 72:1491-501. doi: 10.1097/TA.0b013e318256e000

38. Gutiérrez S, Svahn SL, Johansson ME. Effects of Omega-3 Fatty Acids on Immune Cells. Int J Mol Sci. (2019) 20:5028. doi: 10.3390/ijms20205028

39. Dicarlo M, Teti G, Verna G, Liso M, Cavalcanti E, Sila A, et al. Quercetin exposure suppresses the inflammatory pathway in intestinal organoids from winnie mice. Int J Mol Sci. (2019) 20:5771. doi: 10.3390/ijms20 225771

40. Cianciosi D, Forbes-Hernández TY, Afrin S, Gasparrini M, ReboredoRodriguez P, Manna PP, et al. Phenolic compounds in honey and their associated health benefits: a review. Molecules. (2018) 23:2322. doi: 10.3390/molecules23092322

41. De Santis S, Galleggiante V, Scandiffio L, Liso M, Sommella E, Sobolewski A et al. Secretory leukoprotease inhibitor (Slpi) expression is required for educating murine dendritic cells inflammatory response following quercetin exposure. Nutrients. (2017) 9:706. doi: 10.3390/nu9070706

42. Delvecchio FR, Vadrucci E, Cavalcanti E, De Santis S, Kunde D, Vacca M, et al. Polyphenol administration impairs T-cell proliferation by imprinting a distinct dendritic cell maturational profile. Eur J Immunol. (2015) 45:263849. doi: 10.1002/eji.201545679

43. Cavalcanti E, Vadrucci E, Delvecchio FR, Addabbo F, Bettini S, Liou R, et al. Administration of reconstituted polyphenol oil bodies efficiently suppresses dendritic cell inflammatory pathways and acute intestinal inflammation. PLoS ONE. (2014) 9:e88898. doi: 10.1371/journal.pone.0088898

44. Chen W, Lim CE, Kang HJ, Liu J. Chinese herbal medicines for the treatment of type A H1N1 influenza: a systematic review of randomized controlled trials. PLoS ONE. (2011) 6:e28093. doi: 10.1371/journal.pone.0028093

45. Poon PM, Wong CK, Fung KP, Fong CY, Wong EL, Lau JT, et al. Immunomodulatory effects of a traditional Chinese medicine with potential antiviral activity: a self-control study. Am J Chin Med. (2006) 34:1321. doi: 10.1142/S0192415X0600359X

46. Teng H, Chen L. Polyphenols and bioavailability: an update. Crit Rev Food Sci Nutr. (2019) 59:2040-51. doi: 10.1080/10408398.2018.1437023
47. Leri M, Scuto M, Ontario ML, Calabrese V, Calabrese EJ, Bucciantini M, et al. Healthy effects of plant polyphenols: molecular mechanisms. Int J Mol Sci. (2020) 21:1250. doi: 10.3390/ijms21041250

48. De Santis S, Cavalcanti E, Mastronardi M, Jirillo E, Chieppa M. Nutritional keys for intestinal barrier modulation. Front Immunol. (2015) 6:612. doi: 10.3389/fimmu.2015.00612

49. Corrêa TA, Rogero MM. Polyphenols regulating microRNAs and inflammation biomarkers in obesity. Nutrition. (2019) 59:150-7. doi: 10.1016/j.nut.2018.08.010

50. Alexander M, Hu R, Runtsch MC, Kagele DA, Mosbruger TL, Tolmachova T, et al. Exosome-delivered micrornas modulate the inflammatory response to endotoxin. Nat Commun. (2015) 6:7321. doi: 10.1038/ncomms8321

51. Cione E, La Torre C, Cannataro R, Caroleo MC, Plastina P, Gallelli L. Quercetin, epigallocatechin gallate, curcumin, and resveratrol: from dietary sources to human microRNA modulation. Molecules. (2019) 25:63. doi: 10.3390/molecules25010063

52. Cui J, Zhou B, Ross SA, Zempleni J. Nutrition, microRNAS, and human health. Adv Nutr. (2017) 8:105-12. doi: 10.3945/an.116.013839

53. Cannataro R, Perri M, Gallelli L, Caroleo MC, De Sarro G, Cione E. Ketogenic diet acts on body remodeling and microRNAS expression profile. Microrna. (2019) 8:116-26. doi: 10.2174/2211536608666181126093903

54. Cannataro R, Caroleo MC, Fazio A, La Torre C, Plastina P, Gallelli L, et al. Ketogenic diet and microRNAS linked to antioxidant biochemical homeostasis. Antioxidants. (2019) 8:269. doi: 10.3390/antiox8080269

55. Ling JX, Wei F, Li N, Li JL, Chen LJ, Liu YY, et al. Amelioration of influenza virus-induced reactive oxygen species formation by epigallocatechin gallate derived from green tea. Acta Pharmacol Sin. (2012) 33:153341. doi: 10.1038/aps.2012.80

56. Liu Q, Lu L, Hua M, Xu Y, Xiong H, Hou W, et al. Jiawei-Yupingfeng-Tang, a Chinese herbal formula, inhibits respiratory viral infections in vitro and in vivo. J Ethnopharmacol. (2013) 150:521-8. doi: 10.1016/j.jep.2013.08.056

57. De Santis S, Kunde D, Serino G, Galleggiante V, Caruso ML, Mastronardi $\mathrm{M}$, et al. Secretory leukoprotease inhibitor is required for efficient quercetinmediated suppression of TNF $\alpha$ secretion. Oncotarget. (2016) 7:758009. doi: 10.18632/oncotarget.12415

58. Yahfoufi N, Alsadi N, Jambi M, Matar C. The immunomodulatory and anti-inflammatory role of polyphenols. Nutrients. (2018) 10:1618. doi: 10.3390/nu10111618

59. Imai Y. Role of omega-3 PUFA-derived mediators, the protectins, in influenza virus infection. Biochimi Biophys Acta. (2015) 1851:496-502. doi: 10.1016/j.bbalip.2015.01.006

60. Seidelmann SB, Claggett B, Cheng S, Henglin M, Shah A, Steffen LM, et al. Dietary carbohydrate intake and mortality: a prospective cohort study and meta-analysis. Lancet Public Health. (2018) 3:e41928. doi: 10.1016/S2468-2667(18)30135-X

61. Cena H, Calder PC. Defining a healthy diet: evidence for the role of contemporary dietary patterns in health and disease. Nutrients. (2020) 12:334. doi: 10.3390/nu12020334

62. Salas-Salvadó J., Díaz-López A., Ruiz-Canela M., et al. PREDIMED-Plus investigators. effect of a lifestyle intervention program with energy-restricted mediterranean diet and exercise on weight loss and cardiovascular risk factors: one-year results of the PREDIMED-Plus trial. Diabetes Care. (2018) 42:77788. doi: $10.2337 / \mathrm{dc} 18-0836$

63. Adhikari SP, Meng S, Wu YJ, Mao YP, Ye RX, Wang QZ, et al. Epidemiology, causes, clinical manifestation and diagnosis, prevention and control of coronavirus disease (COVID-19) during the early outbreak period: a scoping review. Infect Dis Poverty. (2020) 9:29. doi: 10.1186/s40249-020-00646-x

Conflict of Interest: The authors declare that the research was conducted in the absence of any commercial or financial relationships that could be construed as a potential conflict of interest.

Copyright (c) 2020 Cena and Chieppa. This is an open-access article distributed under the terms of the Creative Commons Attribution License (CC BY). The use, distribution or reproduction in other forums is permitted, provided the original author(s) and the copyright owner(s) are credited and that the original publication in this journal is cited, in accordance with accepted academic practice. No use, distribution or reproduction is permitted which does not comply with these terms. 